\title{
An early peak in ion channel research
}

To the Editor - The recent experimental demonstration of non-equilibrium switching kinetics in the transitions between clockwise and counterclockwise rotation of the bacterial flagellar motor by Wang et al. ${ }^{1}$ is an elegant illustration of how theoretical results in non-equilibrium thermodynamics can lead to practical biological insight. Citing theoretical work by Tu from $2008^{2}$, they note that the presence of peaked waiting time distributions between switching events is incompatible with detailed balance in the underlying kinetics, showing that the switching process is actively driven by some dissipative process.

This line of argument, as well as the underlying theoretical result, mirrors an early development in the study of ion channels. Peaked waiting time distributions in the current traces of single glutamate-gated channels were reported by Gration et al. in $1982^{3}$, and the connection between peaks and non-equilibrium kinetics was quickly noted for a simple three-state model ${ }^{4}$. A general proof was then given by Kijima and Kijima in $1987^{5}$, whose theorem I is equivalent to equation 1 in ref. 2.
References

1. Wang, F. et al. Nat. Phys. 13, 710-714 (2017).

2. Tu, Y. Proc. Natl Acad. Sci. USA 105, 11737-11741 (2008).

3. Gration, K. A. F., Lambert, J. J., Ramsey, R. L., Rand, R. P. \& Usherwood, P. N. R. Nature 295, 599-601 (1982).

4. Colquhoun, D. \& Hawkes, A. G. in Single-Channel Recording (eds Sakmann, B. \& Neher, E.) 135-174 (Plenum, 1983).

5. Kijima, S. \& Kijima, H. J. Theor. Biol. 128, 423-434 (1987).

\section{Martin Lindén}

Department of Cell and Molecular Biology, Uppsala University, 75236 Uppsala, Sweden.

$\square \quad$ e-mail: martin.linden@icm.uu.se 